\title{
Stress management intervention to enhance adolescent resilience: a randomized controlled trial
}

\author{
Maria-Despoina K. Kallianta ${ }^{1}$, Xrysoula E. Katsira ${ }^{1}$, Artemis K. Tsitsika ${ }^{2}$, Dimitrios Vlachakis ${ }^{3,4,5}$, George \\ Chrousos $^{5}$, Christina Darviri ${ }^{1}$, Flora Bacopoulou ${ }^{5 凶}$
}

${ }^{1}$ Postgraduate Course of Science of Stress and Health Promotion, School of Medicine, National and Kapodistrian University of Athens, Athens, Greece

${ }^{2}$ Adolescent Health Unit, Second Department of Pediatrics, «P. \& A. Kyriakou» Children's Hospital, National and Kapodistrian University of Athens, Athens, Greece

${ }^{3}$ Laboratory of Genetics, Department of Biotechnology, School of Applied Biology and Biotechnology, Agricultural University of Athens, Athens, Greece

${ }^{4}$ Lab of Molecular Endocrinology, Center of Clinical, Experimental Surgery and Translational Research, Biomedical Research Foundation of the Academy of Athens, Greece

${ }^{5}$ University Research Institute of Maternal and Child Health \& Precision Medicine, and UNESCO Chair on Adolescent Health Care, National and Kapodistrian University of Athens, Aghia Sophia Children's Hospital, Athens, Greece

Competing interests: MDKK none; XEK none; AKT none; DV none; GC none; CD none; FB none

\section{Abstract}

The purpose of this study was to examine the effects of an 8-week stress management intervention to enhance resilience and coping techniques and decrease stress in adolescent students. Teenagers, 11 to 17 years old, recruited from two tertiary Adolescent Medicine Centers of the National and Kapodistrian University of Athens, Greece, were randomly assigned into two groups: the stress management group $(n=24)$ and the control group $(n=25)$. Resilience, stress, anxiety, everyday use of social media, school performance and cognitive skills were measured in adolescents of both groups, pre- and post-intervention. Post-intervention, the stress management group had significantly higher resilience scores and school performance self-evaluation scores, lower scores of stress, anxiety and everyday use of social media and better cognitive skills than the control group. Regarding cognitive skills, the stress management group significantly improved the speed of information processing and memory. Adolescents following stress management experienced significantly reduced stress from interacting with teachers/parents, from peer pressure, from school/leisure conflict as well as compulsive behaviours. With respect to resilience, the intervention improved adolescents' individual skills and resources, relationships with primary caregivers, and environmental factors that facilitated the sense of belonging. Future studies of large adolescent samples are required to evaluate the long-term benefits of stress management techniques on adolescents' health and resilience, as well as the need of continued support to preserve these benefits throughout transition to adulthood.

\section{Introduction}

Adolescence is a crucial transition period from childhood to adulthood, during which teenagers experience many challenges that can ultimately affect their health. These challenges can also be referred to as stressors, and can be intrapersonal (e.g., changes in sleeping and eating habits), academic (e.g., increased class workload), interpersonal (e.g., change in social activities) and environmental (e.g., increased screen time) (Ross et al., 1999). Stress is a multidimensional term. In 1970, psychologists
Richard Lazarus and Susan Folkman introduced the concept of perceived stress and defined stress as a transaction between the person and the environment. They argued that when a person feels unable to cope with the demands of the environment, based on personal abilities and skills, then he or she experiences stress (Lazarus and Folkman, 1984). Later, the pediatricianendocrinologist George Chrousos defined stress as the situation of real or perceived threat to homeostasis and described the complex network of behavioural and physiological adaptation responses (Chrousos and Gold,

\section{Article history}

Received: 17 October 2020

Accepted: 28 November 2020

Published: 23 August 2021

(c) 2021 Kallianta et al:; the authors have retained copyright and granted the Journal right of first publication; the work has been simultaneously released under a Creative Commons Attribution Licence, which allows others to share the work, while acknowledging the original authorship and initial publication in this Journal. The full licence notice is available at http://journal.embnet.org. 
1992). Resilience refers to successful adaptation to stress; Flora Bacopoulou and George Chrousos have defined resilience as the ability of the individual to maintain, regain or improve homeostasis - eustasis or hyperstasis - despite adverse life experiences (Bacopoulou and Chrousos, 2013). There are several literature reports of everyday hassles and major stressful life events (natural disasters, poverty, parental divorce, and parental psychopathology) as risk factors for depression, reduced skills and low resilience (Luthar and Zigler, 1991). Resilience is a dynamic process of positive adjustment in the context of significant adversity (Luthar et al., 2000). Resilience can be perceived as the positive response and recovery capacity after major setbacks, excessive demands, and intensely stressful situations. The main feature of resilience is the ability of the individual to return to his / her original level of function after any stressful event (Carver, 1998; O'Leary and Ickovics, 1995; Block and Block, 1980; Block and Kremen, 1996; Lazarus, 1993). Psychologists have studied resilience for years, but nowadays the goal is to enhance resilience before the exposure to a stressful or traumatic event (Everly et al., 2008). Resilience is positively correlated with selfefficacy (Bandura, 1997; 2003), hardiness (Kobasa et al., 1982), internal locus of control (Luthar, 1991; Sandler et al., 2000; Grobet et al., 1995; Wyman, 2003), social skills and social support, adolescent coping skills (WHO, 2004; Jensenet et al., 2006; Everly and Lating, 2002; Steinhardt and Dolbier, 2008) and moderate use of social media. High intelligence, on the other hand, may have a negative impact on resilience according to some investigators. Stress management techniques seem to minimize its negative impact on health and, thus, on resilience and quality of life (Steinhardt and Dolbier, 2008). People with high resilience usually have better ways to manage their stress (coping process) (Frederickson, 2000).

Increasing numbers of researchers have evaluated the effects of stress management programs for students. Such interventions aim at reducing stress and improving health by focusing on relaxation techniques (e.g., diaphragmatic breathing, muscle relaxation, imagery, meditation biofeedback) and cognitivebehavioural strategies which help to identify and change maladaptive thinking (e.g., all- or- none, perfectionism, self-punishing) and behaviour (e.g., alcohol and drug use, smoking, internet addiction). Social support is thought to be a buffer against negative effects of stress and increases resilience. Social support provides a safe environment where individuals are encouraged to share their experiences, thoughts, and feelings.

The purpose of this study was to assess the effects of a stress management technique designed to enhance adolescents' resilience, adaptive coping strategies, and protective factors, as well as decrease maladaptive coping strategies, thinking and behaviours. We hypothesized that adolescent students who attended an 8-week stress management intervention would demonstrate enhanced resilience, coping skills and protective factors, better school performance, as well as decreased maladaptive thinking and behaviours such as time spent on social media, compared to students in the control group.

\section{Materials, Methodologies and Techniques}

\section{Study Sample}

The minimum sample size calculated for each group was $20(\mathrm{~N}=40)$. The sample was recruited from two tertiary Adolescent Medicine Centers of the National and Kapodistrian University of Athens, the Center for Adolescent Medicine and UNESCO Chair on Adolescent Health Care at the "Aghia Sophia" Children's Hospital and the Adolescent Health Unit at the "P. \& A. Kyriakou" Children's Hospital. The study protocol was approved by the Scientific and Ethics Committee of the "Aghia Sophia" Children's Hospital.

\section{Inclusion and exclusion criteria}

Healthy teenagers, 11-17 years old, students able to read and write in Greek, were eligible to participate in the study. Adolescents with diagnosed psychiatric disorders or other diseases, adolescents under psychological or psychiatric treatment, and substance users were excluded.

\section{Study Design}

Adolescents were randomized into two groups, the stress management (SM) group and the control group, based on random numbers derived from a random number generator (random.org). Participants in both groups and their parents or guardians were informed about the program's purposes and signed consent forms prior to study entry.

The intervention comprised health promotion and stress management techniques such as diaphragmatic breathing, progressive muscle relaxation and guided imagery (Steinhardt and Dolbier, 2008). The SM group followed an 8-week stress management program based on the following timetable. Appointments were scheduled with the adolescents.

Stress Management Intervention according to Weeks:

1. Completion of pre-intervention questionnaires. Power point presentation and discussion about stress, healthy lifestyle, and everyday routine. Tips for exercise, systematic walking, disposal of individual pedometers and practical guide on stress management and healthy behaviours in print. Emphasis placed on the implementation of systematic exercise.

2. Power point presentation on application of "Diaphragmatic Breathing" technique, individually or in groups. Learning of diaphragmatic breathing was facilitated by the use of Biofeedback. Recommendation to apply relaxation techniques twice a day (morning and evening). Quality data recorded.

3. Power point presentation on implementation of "Progressive Muscle Relaxation", individually or in 
groups. Learning of progressive muscle relaxation facilitated through a CD. Recommendation to apply the relaxation technique twice a day (morning and evening). Provision of a CD and a booklet of relaxation techniques. Quality data recorded.

4. Power point presentation on "Cognitive Behavioural Therapy". Discussion of the so far implemented techniques and empowerment to continue the program. Quality data recorded.

5. Power point presentation on "Healthy Diet". Empowerment to continue the program. Quality data recorded.

6. Learning and application of the relaxation technique of "Guided imagery", with the use of a CD. Recommendation to apply the technique twice a day (morning and evening). Quality data recorded.

7. Re-check with each participant separately relaxation techniques and encourage healthy behaviours. Quality data recorded.

8. Completion of post-intervention questionnaires. Quality data recorded. Relevant questions and queries were solved, clarifications were given.

Each participant was advised to practice the stress management intervention twice a day; to begin with diaphragmatic breaths and progressive muscle relaxation, and then, after learning the technique of guided imagery, to replace one of the former two techniques, depending on what was best suited for the adolescent. At each session, a qualitative assessment of the changes (benefits) was made, as the individual recorded the results of the intervention. To monitor compliance and solve potential adolescents' problems, a once-a-week appointment was scheduled with participants, who were encouraged to communicate with the researchers through telephone or via e-mail if any problem occurred.

\section{Measures}

Adolescents of both groups, before and after the 8-week intervention, were assessed for their levels of stress, anxiety, resilience and school performance, the frequency of use of social media, as well as their cognitive skills, as follows:

Assessment of Stress levels: Stress levels were assessed with the Adolescent Stress Questionnaire (ASQ) (Darviri et al., 2004; Byrne et al., 2007; Moksnes and Espnes, 2011; De Vriendt et al., 2011). The questionnaire consists of 58 questions about the stress experienced by the teenager in the last year, circling a number from 1 to 5 , based on a 5 -level Likert type scale [ $1=$ No stress (or not applicable to me), 2 = Some Stress, 3 = Moderate Stress, 4 = Enough Stress, 5 = A Lot of Stress].

Assessment of Anxiety levels: Anxiety levels were assessed with the Spence Children's Anxiety Scale (SCAS). The questionnaire consists of 46 questions about the anxiety felt by the teenager. The person is asked to answer the questions based on a 4-stage Likert type scale ( 1 = Never, 2 = Sometimes, 3 = Often, 4 = Always $)$, by circling the appropriate word. The highest score that anyone can get is 114 (Mellon and Moutavelis, 2007).

Assessment of Resilience levels:The Child and Youth Resilience Measure (CYRM) -The Youth version was used to measure adolescents' resilience. The questionnaire consists of 28 questions about the person's personal, social, and interpersonal abilities and his / her level of resilience. The person is asked to answer the questions based on a 3-stage Likert type scale (No, Sometimes, Yes) by circling the appropriate word. Good psychometric properties have been reported with great internal credibility of this tool in other countries (Liebenberg et al., 2012).

Assessment of School Performance: A self-report question about school performance. The question will be answered by the teenager. The question was: "How good do you think you are as a student when you take into account your overall presence in the classroom (homework preparation, classroom participation, performance in tests)?" Respondents will place their answer on a number line from 0 to 10 , with 0 meaning "no good at all" and 10 "excellent".

Assessment of the frequency of use of Social Media: The frequency of social media use was assessed with the self-report question "How many hours do you spend on social networking pages per day on a mobile / tablet / computer?"

Assessment of Cognitive Skills: Cognitive tests assess cognitive skills such as memory, concentration, speed of recall and information processing (Langdon et al., 2012; Sheridan et al., 2006; Stegen et al., 2010; Benedict, 1997). The following cognitive assessment tests were used:

- Normative Symbol Digit Modalities Test (SDMT): The SDMT is a screening instrument. Performance on the SDMT is underpinned by attention, perceptual speed, motor speed and visual scanning. The SDMT consists of a key with two rows, with nine stimulus symbols in the upper row and matched numbers (1-9) in the row below it. The task sequence consists of a series of symbols, each with a blank space underneath that the subject is asked to consecutively fill with the corresponding numbers as fast as possible in 90 seconds after completing a 10 -item practice trial. The score of the test is the number of correct substitutions completed within the time limit, with the maximum score of 110.California Verbal Learning Test-II (CVLTII): The CVLT-II measures both recall and recognition of two lists of words over several immediate- and delayed-memory trials. List A is composed of 16 words, four words each of four semantic categories. An interference list (List B) of 16 words is then presented for one trial. The interference trial is followed by short-delay free-recall and short-delay cued-recall trials for List A. The CVLT-II ends with a new, optional forced-choice recognition trial that is administered approximately 10 minutes following the yes/no recognition trial. 


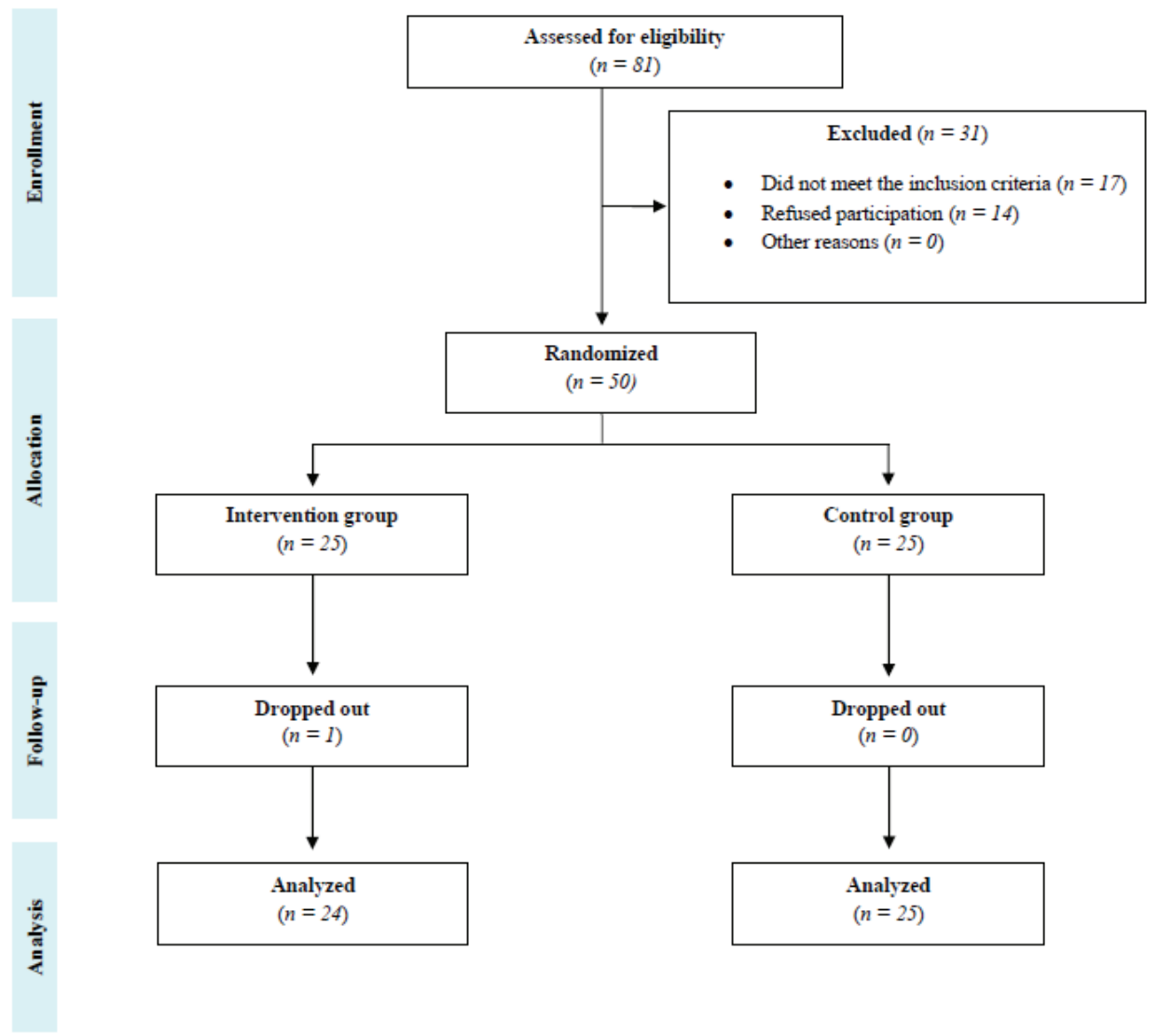

Figure 1. Study flowchart.

- Brief Visuospatial Memory Test-Revised (BVMT-R) (Langdon et al., 2012; Sheridan et al., 2006; Stegen et al., 2010; Benedict, 1997): The BVMT-R was designed as an equivalent multiple test for assessment of visual memory. The BVMT-R comprises six alternate, equivalent forms (Forms 1 through 6). Each form consists of a) 6 geometric figures printed on a $2 \times 3$ array on a separate page of the Recall Stimulus Booklet, and b) 12 recognition items, each printed on a separate page in the Recognition Stimulus Booklet. A manual is required for precise scoring and interpretation, but it is not necessary to have the manual present during the administration of the test.

\section{Statistical analysis}

Longitudinal changes in outcome measures from baseline to 8 weeks (or rate of outcome change) were analysed using linear mixed-effects models with interaction terms for study group and time points. Random intercepts were used for the random effect of each participant in the model using variance components structure (Supplementary models' formula). Means of observed and the adjusted (estimated marginal means for group $\mathrm{x}$ time) outcome changes and their SEs are presented (Table 2). The level of significance was set at 0.05 . Analyses were performed using SPSS version 22.0 (Chicago IL).

\section{Results}

Overall, 81 teens were approached. Of these, 31 were excluded (17 did not meet the inclusion criteria, 14 refused to participate). Fifty adolescents were randomized to the SM intervention group and the control group. One adolescent from the SM group discontinued the followup without a known reason. Finally, 24 adolescents were included in the SM group and 25 in the control group. The study flowchart is presented in Figure 1.

Girls were represented in approximately $70 \%$ in both groups. The median ages in the SM group and the control group were 16.1 and 14.6 years, respectively (Table 1).

Data were compared before and after the intervention, but also between groups, and statistical criteria were used depending on the size and type of the variable (within and between analysis).

Table 1. Demographics of the study sample.

\begin{tabular}{lcc|c} 
Characteristic & $\begin{array}{c}\text { SM Group } \\
(\mathbf{N = 2 4 )}\end{array}$ & $\begin{array}{c}\text { Control group } \\
\mathbf{( N = 2 5 )}\end{array}$ & p value \\
\hline Girls N, (\%) & $17(70.8)$ & $18(72.0)$ & $0.01^{\star}$ \\
\hline $\begin{array}{l}\text { Median Age } \\
\text { (Min,Max), years }\end{array}$ & $16.08(11,17)$ & $14.60(11,17)$ & $<0.001^{*}$ \\
\hline
\end{tabular}

SM: stress management 
Table 2. Results of the Linear Mixed-Effects Models with Random Intercepts for the Rates of Outcome Change.

\begin{tabular}{|c|c|c|}
\hline Characteristic & $\begin{array}{l}\text { b for group } \times \text { time } \\
\text { interaction }(\mathrm{SM} \text { group } \\
\text { vs. Control group }) \pm S E\end{array}$ & p value \\
\hline School performance, self-evaluation & $0.54 \pm 0.17$ & $0.003^{*}$ \\
\hline Hours spent daily on social media & $-1.05 \pm 0.33$ & $0.003^{*}$ \\
\hline Cognitive test- Mathematical test (countdown- right answers) & $1.98 \pm 0.48$ & $0.000^{*}$ \\
\hline Cognitive test- Mathematical test (countdown)- minutes needed to complete & $-6.15 \pm 7.08$ & 0.390 \\
\hline Cognitive test- the three keywords & $0.00 \pm 0.10$ & 1.000 \\
\hline Physical exhaustion & $-0.38 \pm 0.46$ & 0.410 \\
\hline Mental exhaustion & $-1.03 \pm 0.63$ & 0.107 \\
\hline Psychological exhaustion & $-1.31 \pm 0.74$ & 0.085 \\
\hline Cognitive test- Normative Symbol Digit Modalities Test & $18.87 \pm 2.80$ & $0.000^{*}$ \\
\hline Cognitive test- California Verbal Learning Test-II (CVLT-II) & $7.16 \pm 2.20$ & $0.002^{\star}$ \\
\hline Cognitive test- Brief Visuospatial Memory Test Revised (BVMT-R) & $1.02 \pm 1.55$ & 0.515 \\
\hline Stress of teacher/adult interaction (ASQ) & $-4.31 \pm 0.99$ & $0.000^{*}$ \\
\hline Stress of peer pressure (ASQ) & $-2.54 \pm 0.81$ & $0.003^{*}$ \\
\hline Stress of home life (ASQ) & $-3.36 \pm 1.97$ & 0.095 \\
\hline Stress of adult responsibility (ASQ) & $-0.92 \pm 0.49$ & 0.065 \\
\hline Stress of romantic relationship (ASQ) & $0.42 \pm 0.83$ & 0.619 \\
\hline Stress of school attendance (ASQ) & $-1.22 \pm 0.74$ & 0.108 \\
\hline Stress of school/leisure conflict (ASQ) & $-3.05 \pm 1.08$ & $0.007^{*}$ \\
\hline Stress of school performance (ASQ) & $-1.37 \pm 0.76$ & 0.077 \\
\hline Stress of financial pressure (ASQ) & $-0.38 \pm 0.69$ & 0.585 \\
\hline Panic disorder and agoraphobia (SCAS) & $-0.86 \pm 0.81$ & 0.292 \\
\hline Generalized anxiety disorder (SCAS) & $-0.58 \pm 0.78$ & 0.462 \\
\hline Separation anxiety disorder (SCAS) & $-0.76 \pm 0.54$ & 0.166 \\
\hline Physical injury fears (SCAS) & $-0.46 \pm 0.65$ & 0.483 \\
\hline Social phobia (SCAS) & $-0.46 \pm 0.66$ & 0.486 \\
\hline Compulsive behaviour (SCAS) & $-1.14 \pm 0.36$ & $0.002^{*}$ \\
\hline Individual capacities/resources (CYRM) & $1.74 \pm 0.56$ & $0.003^{*}$ \\
\hline Relationship with primary caregiver (CYRM) & $1.16 \pm 0.43$ & $0.010^{*}$ \\
\hline Contextual factors that facilitate the sense of belonging (CYRM) & $1.20 \pm 0.45$ & $0.011^{\star}$ \\
\hline
\end{tabular}

SM: stress management, SE: standard error, ASQ: Adolescent Stress Questionnaire, SCAS: Spence Children's Anxiety Scale, CYRM: Child and Youth Resilience Measure

Table 2 presents the results of mixed effects models concerning changes over time.

Patients in the SM intervention group demonstrated statistically significant reductions in the hours spent daily on social media, in stress (ASQ) and in anxiety (SCAS), and significant increases in resilience (CYRM) and in self-evaluation of school performance. More specifically, in terms of the adolescents' stress, statistically significant lower scores were recorded in stress derived from teacher/adult interaction, from peer pressure and in stress derived from school/leisure conflict. Statistically significant lower scores were also recorded in the compulsive behaviour, assessed by SCAS. Finally, resilience assessed by CYRM showed higher scores in the individual abilities/resources, in the relationship with the primary caregiver and in the factors facilitating the sense of belonging. In terms of cognitive functions, statistically significant differences were noted in the mathematical test (countdown), in the Normative Symbol Digit Modalities Test and in the California Verbal Learning Test-II (CVLT-II).

As shown in Table 3, 8.3-16.7\% of the adolescents exhibited significant score changes according to the Reliable Change Index (RCI) used for the questionnaires. Based on the Number Needed to Treat (NNT) values the strongest effect was noted for processing speed as assessed by the Normative Symbol Digit Modalities Test. 
Table 3. Number of individuals with beneficial significant score changes according to the Reliable Change Index and the corresponding Number Needed to Treat (NNT) values.

\begin{tabular}{llll}
\hline Characteristic (Cronbachs' alpha before, after) & SM group N (\%) & Control group N (\%) & NNT \\
\hline School performance- self-evaluation & $0(0)$ & $0(0)$ & - \\
\hline Hours spent daily on social media & $1(4.2)$ & $0(0)$ & 24 \\
\hline Cognitive test- Mathematical test (countdown- right answers) & $1(4.2)$ & $0(0)$ & 24 \\
\hline Cognitive test- Normative Symbol Digit Modalities Test & $4(16.7)$ & $0(0)$ & 6 \\
\hline Cognitive test- California Verbal Learning Test-II (CVLT-II) & $0(0)$ & $0(0)$ & - \\
\hline Stress of teacher/ adult interaction & $1(4.2)$ & $0(0)$ & - \\
\hline Stress of peer pressure & $0(0)$ & $0(0)$ & - \\
\hline Stress of school/leisure conflict & $0(0)$ & $0(0)$ & - \\
\hline Compulsive behaviour & $0(0)$ & $0(0)$ & 12 \\
\hline Individual capacities/ resources & $2(8.3)$ & $0(0)$ & 24 \\
\hline Relationship with primary caregiver & $1(4.2)$ & $0(0)$ & - \\
\hline Contextual factors that facilitate the sense of belonging & $0(0)$ & $0(0)$ & \\
\hline
\end{tabular}

SM: stress management, NNT: number needed to treat

\section{Discussion}

The aim of this research was to examine whether adolescents following stress management techniques for 8 weeks could improve their resilience, their ability to cope and recover after adversities. This would help them manage more effectively their stress of school and perform better, adopt a healthier lifestyle i.e. reduce hours in social media, and build stronger relationships. The study results demonstrated statistically significant differences between the SM intervention group and the control group in several areas. More specifically, the SM group (vs. the control group) experienced improvement in school performance (by self-evaluation) and reduced the hours spent on social media. Regarding cognitive skills, the SM group significantly improved the speed of information processing and memory. Stress management techniques seem to have helped in reducing adolescents' stress from interacting with teachers/parents, from peer pressure, from school/leisure conflict as well as compulsive behaviours. Adolescents in the SM group improved their resilience, their individual skills and resources, their relationship with primary caregivers, and the environmental factors that facilitated the sense of belonging.

The teenagers who participated in the SM group significantly reduced the stress they experienced in relation to their social environment. This concept is inextricably linked to the concept of resilience. Selfefficacy and support from the social environment (as well as the teen's ability to ask for this support without being ashamed or afraid) are important to enhance resilience (Werner and Smith, 1982; Fergus and Zimmerman, 2005; Zolkoski and Bullock, 2012). On the other hand, academic success/performance is also a very important factor which enhances adolescents' resilience, according to the protective factor model of resilience (Fergus and Zimmerman, 2005; Zolkoski and Bullock, 2012). Daily adversities in adolescent life can be offset by other buffers such as academic performance and supportive social environment. Similarly, protective or reinforcing factors for young people can also be considered the improvement of cognitive abilities, such as the speed of processing information and memory but also the improvement of individual capacities and resources, the relationship with primary caregivers and other environmental factors that facilitate the sense of belonging. All the aforementioned variables were significantly improved in the intervention group, as already mentioned. Furthermore, personal self-control (Fergus and Zimmerman, 2005; Zolkoski and Bullock, 2012) has an impact on everyday routine (mainly in eating and sleeping) and is very important in enhancing resilience. The reduction of stress in many aspects of the teen's daily routine, is very important and is being pursued by many programs trying to enhance adolescents' resistance and resilience against adversities (Daining and DePanfilis, 2007; Steinhardt and Dolbier, 2008; Abolghasemi and Varaniyaba, 2010).

The adolescents who participated in the SM intervention group experienced statistically significant improvement in their routine, since they improved the self-evaluation of their school performance and they reduced the hours spent daily on social media. Cognitive skills also recorded significantly higher scores. The relationship between stress and cognitive functions has been highlighted and fully elucidated by a very large volume of literature (McEwen and Sapolsky, 1995; Pechtel and Pizzagalli, 2011; Yuen et al., 2012). Lupien et al. (Lupien et al., 2009) showed that chronic exposure to stress hormones during the prenatal period, infancy, childhood, adolescence, adulthood, or old age, has an 
impact on brain structures involved in cognition and mental health. In this study, it appeared, also, that stress management in adolescents helped them improve some of their cognitive functions.

There are some limitations that must be considered before interpreting the results of this study. The sample was small (a total of 49 adolescents, mainly girls), therefore, the results cannot be generalized to the broader adolescent population. The study included self-reported questionnaires and thus, a subjective view of the sample responses. Furthermore, there was no objective measurement of compliance to the stress management techniques during the program despite the frequent feedback from the adolescents. Finally, it is not known whether the benefits were maintained after the completion of the program, as there was no followup. However, despite the short duration of the program, several beneficial outcomes were reported.

Future studies of large adolescent samples are required to evaluate the long-term benefits of stress management techniques on adolescents' health and resilience, as well as the need of continued support to preserve these benefits throughout transition to adulthood.

\section{Key Points}

- An 8-week stress management intervention enhanced resilience in adolescents.

- Stress, compulsive behaviours and social media time were reduced post-intervention.

- Adolescents experienced improvement in school performance and cognitive skills.

\section{References}

1. Abolghasemi A, Taklavi S (2010) Resilience and perceived stress predictors of life satisfaction in the students of success and failure. Procedia - Social and Behavioral Sciences 5, 748-752. http://dx.doi.org/10.1016/j.sbspro.2010.07.178

2. Bacopoulou F, Chrousos $G$ (2013) Health risk behaviors and resilience in adolescence. In: Antoniou AS and Kirkcaldy BD (Eds). Education, Family and Child \& Adolescent Health. DIADRASSI Publications, pp. 141-154.

3. Bandura A (1997) Self-efficacy: The exercise of control. New York: Freeman.

4. Beasley M, Thompson T, Davidson J (2003) Resilience in response to life stress: the effects of coping style and cognitive hardiness. Pers Individ Dif 34, 77-95. http://dx.doi.org/10.1016/S01918869(02)00027-2

5. Benedict RHB (1997) Brief Visuospatial Memory Test Revised professional manual. Odessa, FL: Psychological Assessment Resource.

6. Block JH, Block J (1980) The role of ego-control and egoresiliency in the origination of behavior. In: Collings WA (Ed) The Minnesota Symposia on Child Psychology. Hillsdale, NJ Erlbaum, Vol. 13, pp. 39-101.

7. Block J, Kremen AM (1996) IQ and ego-resiliency: Conceptual and empirical connections and separateness. Journal of Personality and Social Psychology 70, 349-361. http://dx.doi. org/10.1037/0022-3514.70.2.349.

8. Byrne DG, Davenport SC, Mazanov J (2007) Profiles of adolescent stress: The development of the adolescent stress questionnaire (ASQ). Journal of Adolescence 30 (3), 393-416. http://dx.doi. org/10.1016/j:adolescence.2006.04.004
9. Carver CS (1998) Resilience and thriving: issues, models, and linkages. J Soc Issues 54, 245-266. http://dx.doi. org/10.1111/0022-4537.641998064

10. Chrousos GP, Gold PW (1992) The concepts of stress and stress system disorders. JAMA 267 (9), 1244-1252. http:/! dx.doi:10.1001/jama.1992.03480090092034

11. Daining C, DePanfilis D (2007) Resilience of youth in transition from out-of-home care to adulthood. Children and Youth Services Review 29, 1158-1178. http://dx.doi.org/10.1016/j. childyouth.2007.04.006

12. Darviri C, Legaki P, Chatzioannidou P, Gnardellis Ch, Kraniotou Ch et al. (2014) Adolescent Stress Questionnaire: Reliability and validity of the Greek version and its description in a sample of high school (lyceum) students. Journal of Adolescence 37, 13731377. http://dx.doi.org/10.1016/j.adolescence.2014.10.003

13. De Vriendt T, Clays E, Moreno LA, Bergman P, VicenteRodriguez G et al. (2011) Reliability and validity of the Adolescent Stress Questionnaire in a sample of European adolescents - the HELENA study. BMC Public Health 11, 717. http://dx.doi. org/10.1186/1471-2458-11-717

14. Everly GS Jr, Lating JM (2002) A clinical guide to the treatment of the human stress response. NY: Plenum.

15. Everly G, Welzant V, Jacobson J (2008) Resistance and resilience: The final frontier in traumatic stress management. International Journal of Emergency Mental Health 4(10), 261-270.

16. Fergus S, Zimmerman MA (2005) Adolescent resilience: A framework for understanding healthy development in the face of risk. Annu. Rev Public Health 26, 399-419. http://dx.doi. org/10.1146/annurev.publhealth.26.021304.144357

17. Frederickson BL (2000) Cultivating positive emotions to optimize health and well-being. Prevention \& Treatment 3 (1). http://! dx.doi.org/10.1037/1522-3736.3.1.31a

18. Grob A, Flammer A, Wearing AJ (1995). Adolescents' perceived control: Domain specificity, expectations, and appraisal. Journal of Adolescence 18, 403-425. http://dx.doi.org/10.1006/ jado.1995.1030

19. Jensen LW, Decker L, Andersen MM (2006) Depression and health-promoting lifestyles of persons with mental illnesses. Issues of Mental Health Nursing 27, 617-634. http://dx.doi. org/10.1080/01612840600642919

20. Kobasa SC, Maddi SR, Kahn S (1982) Hardiness and health: A prospective study. Journal of Personality and Social Psychology 42, 168-177. http://dx.doi.org/10.1037/0022-3514.42.1.168

21. Langdon DW, Amato MP, Boringa J, Brochet B, Foley $\mathrm{F}$ et al. (2012) Recommendations for a brief international cognitive assessment for multiple sclerosis (BICAMS). Multiple Sclerosis 18 (6), 891-898. http://dx.doi.org/10.1177/1352458511431076

22. Lazarus R, Folkman S (1984) Stress, Appraisal, and Coping. New York: Springer.

23. Lazarus RS (1993) From psychological stress to the emotions: A history of changing outlooks. Annual Review of Psychology 44, $1-21$.

24. Liebenberg L, Ungar M, Van de Vijver F (2012) Validation of the Child and Youth Resilience Measure-28 (CYRM-28) among Canadian Youth. Research on Social Work Practice 22b(2), 219226. http://dx.doi.org/10.1177/1049731511428619

25. Lupien SJ, McEwen BS, Gunnar MR, Heim C (2009) Effects of stress throughout the lifespan on the brain, behaviour and cognition. Nature Reviews Neuroscience 10, 434- 445.

26. Luthar SS, Zigler E (1991) Vulnerability and competence: a review of research on resilience in childhood. Am J Orthopsychiatry 61(1), 6-22. http://dx.doi.org/10.1037/h0079218

27. Luthar SS (1991) Vulnerability and Resilience: A study of highrisk adolescents. Child Dev 62 (3), 600-616. http://dx.doi. org/10.1111/j.1467-8624.1991.tb01555.x

28. Luthar SS, Cicchetti D, Becker B (2000) The construct of resilience: a critical evaluation and guidelines for future work. Child Development 71 (3), 543-562. http://dx.doi.org/10.1111/1467-. 8624.00164 
29. McEwen BS, Sapolsky RM (1995) Stress and cognitive function. Current Opinion in Neurobiology 5 (2), 205-216. http://dx.doi. org/10.1016/0959-4388(95)80028-X

30. Mellon RC, Moutavelis AG (2007) Structure, developmental course, and correlates of children's anxiety disorderrelated behavior in a Hellenic community sample. Journal of Anxiety Disorders 21, 1-21. http://dx.doi.org/10.1016/i janxdis.2006.03.008

31. Moksnes UK, Espnes GA (2011) Evaluation of the Norwegian version of the Adolescent Stress Questionnaire (ASQ-N): factorial validity across samples. Scandinavian Journal of Psychology 52 (6), 601-608. http://dx.doi.org/10.1111/j.14679450.2011.00907.x

32. O'Leary VE, Ickovics JR (1995) Resilience and thriving in response to challenge: an opportunity for a paradigm shift in women's health. Women's Health Res Gender Behav Policy 1, 121-142.

33. Pechtel P, Pizzagalli DA (2011) Effects of Early Life Stress on Cognitive and Affective Function: An Integrated Review of Human Literature. Psychopharmacology (Berl) 214 (1), 55-70. http://dx.doi.org/10.1007/s00213-010-2009-2

34. Ross SE, Niebling BC, Heckert TM (1999) Sources of stress among college students. Coll Stud J 33, 312- 317.

35. Sandler IN, Kim-Bae L, MacKinnon DP (2000) Coping and appraisal as mediators of the effects of locus of control beliefs on psychological symptoms for children of divorce. Journal of Clinical Child Psychology 29, 336-347. http://dx.doi org/10.1007/s10826-015-0338-9
36. Sheridan LK, Fitzgeralda HE, Adams KM, Nigg JT, Martel MM et al. (2006) Normative symbol digit modalities test performance in a community-based sample. Archives of Clinical Neuropsychology 21, 23-28.

37. Stegen S, Stepanov I, Cookfair D, Schwartz E, Hojnacki D et al. (2010) Validity of the California Verbal Learning Test-II in Multiple Sclerosis. The Clinical Neuropsychologist 24 (2), 189202.

38. Steinhardt M, Dolbier C (2008) Evaluation of a resilience intervention to enhance coping strategies and protective factors and decrease symptomatology. Journal of American College Health 56 (4), 445-453.

39. Werner EE, Smith RS (1982) Vulnerable but invincible: A study of resilient children. New York: McGraw-Hill.

40. World Health Organization (WHO) (2004) Proceedings of the 2004 Conference on Prevention of Mental Disorders: Effective Interventions and Options. Geneva: World Health Organization.

41. Wyman PA (2003) Emerging perspectives on context specificity of children's adaptation and resilience. In: Luthar SS (ed.). Resilience and Vulnerability: Adaptation in the Context of Childhood Adversities. Cambridge University Press, New York, pp. 293-317.

42. Yuen EY, Wei J, Liu W, Zhong P, Li X et al. (2012) Repeated stress causes cognitive impairment by suppressing glutamate receptor expression and function in prefrontal cortex. Neuron 73, 962977. http://dx.doi.org/10.1016/j.neuron.2011.12.033

43. Zolkoski SM, Bullock LM (2012) Resilience in children and youth: A review. Children and Youth Services Review 34, 2295-2303. http://dx.doi.org/10.1016/j.childyouth.2012.08.009 